\title{
Analytic solution of Hubbell's model of local community dynamics
}

\author{
Alan J. McKane, ${ }^{\mathrm{a}, *}$ David Alonso, ${ }^{\mathrm{b}, \mathrm{c}}$ and Ricard V. Soléb,d \\ ${ }^{a}$ Theoretical Physics Group, Department of Physics and Astronomy, University of Manchester, Manchester M13 9PL, UK \\ ${ }^{\mathrm{b}}$ Complex Systems Lab, Universitat Pompeu Fabra, Dr Aiguader 80, 08003 Barcelona, Spain \\ ${ }^{\mathrm{c}}$ Department of Ecology, Facultat de Biologia, Universitat de Barcelona, Diagonal 645, 08045 Barcelona, Spain \\ ${ }^{\mathrm{d}}$ Santa Fe Institute, 1399 Hyde Park Road, NM 87501, USA
}

Received 16 April 2003

\begin{abstract}
Recent theoretical approaches to community structure and dynamics reveal that many large-scale features of community structure (such as species-rank distributions and species-area relations) can be explained by a so-called neutral model. Using this approach, species are taken to be equivalent and trophic relations are not taken into account explicitly. Here we provide a general analytic solution to the local community model of Hubbell's neutral theory of biodiversity by recasting it as an urn model, i.e. a Markovian description of states and their transitions. Both stationary and time-dependent distributions are analysed. The stationary distribution - also called the zero-sum multinomial - is given in closed form. An approximate form for the time-dependence is obtained by using an expansion of the master equation. The temporal evolution of the approximate distribution is shown to be a good representation for the true temporal evolution for a large range of parameter values.
\end{abstract}

(C) 2003 Elsevier Inc. All rights reserved.

Keywords: Community dynamics; Hubbell's neutral theory; Abundance distribution; Zero-sum multinomial; Analytic solution

\section{Introduction}

Understanding the global patterns of biodiversity and its dynamics at different time scales remains a great challenge for ecological science (Rosenzweig, 1995; Wilson, 2003). One of the key features that defines community structure is the relation between range and abundance. How the community structure develops in time and how species are spatially distributed largely define the field of macroecology (Brown, 1995). In this context, an important step to unify biogeography and biodiversity has been achieved by Hubbell (Hubbell, 2001; Bell, 2001) through the formulation of a neutral theory.

The mathematical framework employed by Hubbell allows for speciation processes to be integrated with the MacArthur-Wilson theory of island biogeography. In this way, the neutral theory predicts some universal features that can be tested by direct analysis of speciesabundance distributions and other large-scale measurements. In Hubbell's theory, two key quantities largely

${ }^{*}$ Corresponding author. Fax: +44-161-275-4218.

E-mail address: alan.mckane@man.ac.uk (A.J. McKane). determine the steady-state distributions of species richness (as well as relative species abundances on local and large geographic scales). These two parameters are the so-called biodiversity number and the immigration (dispersal) rate. Under Hubbell's assumptions, the ecological properties of every individual in the population are assumed to be identical.

In a neutral model of this type, individuals compete for the same pool of resources, but chance events are responsible for the identity of the final winner(s). The dynamics of each species is thus path-dependent and a Markovian description of their time evolution is appropriate. Under the assumption of a balance between birth, death and immigration rates, the neutral theory is able to reproduce the quantitative patterns of species distributions that are well known from the ecological literature. It also permits the generation of several non-trivial and testable quantitative predictions about biodiversity and biogeography. In particular, the theory predicts that rare species would typically be recent in terms of their origination. In relation to conservation biology, a neutral community in which species are essentially equal would be very fluid, with frequent replacements. If true, protected areas should be 
larger than those expected for stable communities with species closely adapted to given niches.

Formally, Hubbell's theory is the ecological analog to the neutral theory of genetic drift in genetics (Kimura, 1983; Ewens, 1972; Karlin and McGregor, 1972). Early attempts to incorporate the neutral approach from population genetics (Caswell, 1976; Hubbell, 1997) mainly highlighted the relevance of drift in community dynamics, providing evidence for a global view of ecosystems in which competitive forces, ecological niches, and even trophic interactions could be ignored in the pursuit of a better understanding of biodiversity dynamics. More recent work incorporated these ideas in an explicit way (Hubbell, 1997; Solé and Alonso, 1998) and Hubbell's recent book provides an extensive, unifying account of these (Hubbell, 2001). The starting point of neutral models is a random community that evolves towards an equilibrium state under a stochastic birth- and death-process incorporating dispersal. At high immigration rates, Hubbell's theory predicts a logseries distribution for the abundance of species in the local community, while when the immigration coupling between the metacommunity and the local community is lower, a lognormal-like shape is obtained for this distribution. Within Hubbell's approximation, these distributions are shown to be particular cases of what he denotes as the zero-sum multinomial (Hubbell, 2001).

Hubbell's model for local communities is similar to that proposed in Solé et al. (2000) and analysed in McKane et al. (2000). There we took advantage of a mean field argument to find an analytical form for the stationary distribution for the probability of finding species having an abundance of $n$ individuals. In addition, we studied in detail its time behaviour using different approximations. Furthermore, our simplified approach based on this mean field argument allowed us to recover the scaling relationship between the fraction of links actualised and the number of species in a community - the so-called $C^{*}-S$ relation, and gave conditions in which such a relation arose.

Within Hubbell's mathematical framework the dynamical stochastic models were numerically solved and the equilibrium properties analysed. In this paper we present an analytic, general solution of Hubbell's model for the local community dynamics, that provides the stationary species-abundance distributions together with the time evolution from the initial state towards the stationary distribution.

\section{Formulation of the theory}

Hubbell's theory concerns populations on two scales: local communities and regional metacommunities. To explain the model and derive the equations in the simplest possible way, we will use the language of urn models (Feller, 1968; Johnson and Kotz, 1977). This is a natural description when the stochastic dynamics in one time step only depends on the state of the system at the beginning of the time step (in other words is a Markov process). It also provides us with a concrete picture of the process which aids the derivation of the governing equation for the model.

We begin by considering the model in a limit where the two levels of description are uncoupled. This allows us to focus only on the local community. We assume that there are $N_{i}$ individuals of species $i$ in the local community, with the total number of individuals of all species being $J$, that is, $J=\sum_{i=1}^{r} N_{i}$ where $r$ is the total number of species. The model is defined by picking one individual at random from the local community, killing it, and then replacing it by an individual also drawn from the local community. In terms of the associated model this corresponds to having $N_{i}$ balls of colour $i(i=1, \ldots, r)$ in the urn. If we focus on one particular colour, $j$, the probability that the number of balls will decrease from $N_{j}$ to $N_{j}-1$ during one time step is

$W\left(N_{j}-1 \mid N_{j}\right)=\frac{N_{j}}{J} \frac{\left((J-1)-\left(N_{j}-1\right)\right)}{J-1}$,

since a ball of colour $j$ must be discarded and one of any other colour replaced for such a transition to occur. On the other hand, the probability that the number of balls will increase from $N_{j}$ to $N_{j}+1$ requires that a ball of any other colour but $j$ must be discarded, and one of colour $j$ be replaced. Therefore,

$W\left(N_{j}+1 \mid N_{j}\right)=\frac{\left(J-N_{j}\right)}{J} \frac{N_{j}}{J-1}$.

The whole point of the model, however, is to couple local communities and regional metacommunities. This is achieved by choosing a replacement ball from the urn only $(1-m)$ of the time. For the rest of the time it is chosen from outside the urn. The probability of picking a ball of colour $j$ from this external source is defined to be $P_{j}$, and corresponds to assuming that the replacement individual comes from the regional metacommunity where species $i$ has a relative abundance of $P_{i}$. The transition probabilities (1) and (2) now read

$$
\begin{aligned}
W\left(N_{j}-1 \mid N_{j}\right)= & (1-m) \frac{N_{j}}{J} \frac{\left(J-N_{j}\right)}{J-1} \\
& +m \frac{N_{j}}{J}\left(1-P_{j}\right)
\end{aligned}
$$

and

$$
\begin{aligned}
W\left(N_{j}+1 \mid N_{j}\right)= & (1-m) \frac{\left(J-N_{j}\right)}{J} \frac{N_{j}}{J-1} \\
& +m \frac{\left(J-N_{j}\right)}{J} P_{j} .
\end{aligned}
$$

The change in the probability that there are $N_{j}$ balls in the urn from time $t$ to the time after one time step has elapsed consists of four contributions. Two of these 
correspond to an increase in this probability (due to transitions from $\left(N_{j}-1\right)$ and $\left(N_{j}+1\right)$ to $\left.N_{j}\right)$ and two to a decrease (due to transitions from $N_{j}$ to $\left(N_{j}+1\right)$ and $\left.\left(N_{j}-1\right)\right)$. The balance equation showing this change is

$$
\begin{aligned}
\Delta P\left(N_{j}, t\right)= & W\left(N_{j} \mid N_{j}-1\right) P\left(N_{j}-1, t\right) \\
& +W\left(N_{j} \mid N_{j}+1\right) P\left(N_{j}+1, t\right) \\
- & \left\{W\left(N_{j}+1 \mid N_{j}\right)+W\left(N_{j}-1 \mid N_{j}\right)\right\} \\
& \times P\left(N_{j}, t\right) .
\end{aligned}
$$

Compared with the long time scales we are interested in - during which many transitions will take place - the step size is very small, and we may take the limit in which $\Delta P\left(N_{j}, t\right) \rightarrow d P\left(N_{j}, t\right) / d t$. The resulting equation is a master equation for the probability $P\left(N_{j}, t\right)$ (Van Kampen, 1981; Gardiner, 1985). Some care is needed with the boundary conditions on this equation: clearly the cases $N_{j}=0$ and $J$ are special cases since there can be no transitions which reduce $N_{j}$ in the former case or which increase $N_{j}$ in the latter case. One possibility is to write two separate equations for these special cases. However, there is no need for this if we first observe that some of these conditions are natural consequences of the form of the transition probabilities. For example, the expressions in (3) and (4) are both zero if $N_{j}=0$ and $J$, respectively. So as long as we agree to impose the formal definitions $W(0 \mid-1)=0$ and $W(J \mid J+1)=0$ the same master equation may be used for all states. In addition, an initial condition needs to be imposed to complete the specification of the problem. Typically, the number of individuals in the local community at $t=0$ will be given: $P\left(N_{j}, 0\right)=\delta_{N_{j}, N_{j, 0}}$.

The mathematical formulation of Hubbell's theory described above can be directly mapped on to another dynamical model of a multispecies community which we introduced a few years ago (Solé et al., 2000, 2002; McKane et al., 2000). In this case though, the nature of the interaction depends on the "score" between one species and another, and a form of mean field theory had to be used in order to describe the dynamics by such a straightforward dynamics. In terms of the notation we have used above $-N$ denoting the number of individuals of a particular species and $J$ denoting the total number of individuals of all species - the transition probabilities of this model are (Solé et al., 2000; McKane et al., 2000):

$W(N+1 \mid N)=C^{*}(1-\mu) \frac{N}{J}\left(\frac{J-N}{J-1}\right)+\frac{\mu}{S} \frac{J-N}{J}$

and

$$
\begin{aligned}
W(N-1 \mid N)= & C^{*}(1-\mu) \frac{N}{J}\left(\frac{J-N}{J-1}\right) \\
& +\frac{\mu}{S}(S-1) \frac{N}{J} .
\end{aligned}
$$

Here $\mu$ is the fraction of the time that replacing of one species by another can happen by chance, and not because the replacement individual belongs to a species which has a positive score against the first. It clearly maps into $m$. The other constants are $S$, the number of species, and $C^{*}$, a parameter related to the degree of connectivity of the matrix of scores between the species. The precise form of the mapping is $C^{*}=1$ and $P_{j}=$ $S^{-1}$.

Since we have analysed this model extensively (McKane et al., 2000) we may simply deduce expressions for quantities of interest in the Hubbell theory by setting $C^{*}=1, S=P_{j}^{-1}$ and $\mu=m$.

\section{Stationary state}

The most straightforward questions we can investigate concern the nature of the stationary state of the theory. Let us begin by introducing the abbreviations

$$
\begin{aligned}
r_{N_{j}} & \equiv W\left(N_{j}-1 \mid N_{j}\right) \\
& =\frac{N_{j}}{J}\left[(1-m) \frac{\left(J-N_{j}\right)}{J-1}+m\left(1-P_{j}\right)\right]
\end{aligned}
$$

and

$$
\begin{aligned}
g_{N_{j}} & \equiv W\left(N_{j}+1 \mid N_{j}\right) \\
& =\frac{\left(J-N_{j}\right)}{J}\left[(1-m) \frac{N_{j}}{J-1}+m P_{j}\right] .
\end{aligned}
$$

The master equation now reads

$$
\begin{aligned}
\frac{d P\left(N_{j}, t\right)}{d t}= & r_{N_{j}+1} P\left(N_{j}+1, t\right)+g_{N_{j}-1} P\left(N_{j}-1, t\right) \\
& -\left\{r_{N_{j}}+g_{N_{j}}\right\} P\left(N_{j}, t\right) .
\end{aligned}
$$

The stationary probability distribution, $P_{s}\left(N_{j}\right)$, is determined by setting $d P\left(N_{j}, t\right) / d t=0$. This gives

$$
\begin{aligned}
& r_{N_{j}+1} P_{s}\left(N_{j}+1\right)-g_{N_{j}} P_{s}\left(N_{j}\right) \\
& \quad=r_{N_{j}} P_{s}\left(N_{j}\right)-g_{N_{j}-1} P_{s}\left(N_{j}-1\right) .
\end{aligned}
$$

This is true for all $N_{j}$, which implies that $r_{N_{j}} P_{s}\left(N_{j}\right)$ $g_{N_{j}-1} P_{s}\left(N_{j}-1\right)=I$, where $I$ is a constant. Applying the boundary condition at $N_{j}=0$, we find that $I=0$ and therefore

$$
r_{N_{j}+1} P_{s}\left(N_{j}+1\right)=g_{N_{j}} P_{s}\left(N_{j}\right), \quad N_{j}=0,1, \ldots, J .
$$

To solve this equation, let us first assume that $m \neq 0$. Then the $r_{N_{j}}$ and $g_{N_{i}}$ given by (8) and (9) are all non-zero and we can solve (12) by iteration to obtain

$P_{s}\left(N_{j}\right)=\frac{g_{N_{j}-1} g_{N_{j}-2 \ldots g} g_{0}}{r_{N_{j}} r_{N_{j}-1} \ldots r_{1}} P_{s}(0), \quad N_{j}=1, \ldots, J$.

The constant $P_{S}(0)$ can be determined from the normalisation condition

$$
\sum_{N_{j}=0}^{J} P_{S}\left(N_{j}\right)=P_{S}(0)+\sum_{N_{j}>0} P_{S}\left(N_{j}\right)=1 .
$$

To simplify the algebra let us introduce some new notation for various combinations of parameters which 
naturally appear in the solution of the model. We write the transition probabilities as

$r_{N_{j}}=\frac{(1-m)}{J(J-1)} N_{j}\left(N_{j}^{*}-N_{j}\right)$

and

$g_{N_{j}}=\frac{(1-m)}{J(J-1)}\left(J-N_{j}\right)\left(N_{j}+P_{j}^{*}\right)$,

where

$P_{j}^{*}=\frac{m(J-1)}{(1-m)} P_{j} \quad$ and $\quad N_{j}^{*}=\left(\frac{J-m}{1-m}\right)-P_{j}^{*}$.

Substituting the expressions (15) and (16) into (13) gives an explicit representation for the $P_{s}\left(N_{j}\right)$ in terms of $P_{s}(0)$. An expression for $P_{s}(0)$ itself can be obtained by performing the finite sum which appears in (14). This sum can be performed analytically using properties of Jacobi polynomials (Abramowitz and Stegun, 1965). Alternatively, the mapping into the model defined by (6) and (7) can be used since the result for the $P_{s}$ is known in this case (Solé et al., 2000; McKane et al., 2000). One finds (see McKane et al., 2000, for details of the derivation):

$P_{s}\left(N_{j}\right)=\left(\begin{array}{c}J \\ N_{j}\end{array}\right) \frac{B\left(N_{j}+P_{j}^{*}, N_{j}^{*}-N_{j}\right)}{B\left(P_{j}^{*}, N_{j}^{*}-J\right)}$,

where $B(a, b)=\Gamma(a) \Gamma(b) / \Gamma(a+b)$ is the beta-function.
It is interesting to note that in the case $m=0$, where the local community is decoupled from the regional metacommunity, $g_{0}=0$, and so from (12), since $r_{1} \neq 0$, it follows that $P_{s}(1)=0$. In fact, since $r_{N_{j}} \neq 0$ for $0<N_{j}<J$, we see from (12) that $P_{s}\left(N_{j}\right)=0$ for all $0<N_{j}<J$. So with no interaction with the regional metacommunity, species $j$ either disappears or becomes the only species there is in the local community. Therefore, some degree of coupling is vital for biodiversity.

In Fig. 1, we have computed the stationary distribution for different parameter values and sizes of the system. The relative species-abundance distribution predicted to occur in local communities - the zero-sum multinomial - by the unified theory of Hubbell can be readily computed even for large community sizes using the analytic formula (18).

\section{Time dependence}

Together with universal features displayed by the stationary patterns observed in mature communities, some common features are also observed when looking at how diversity develops in time. When an empty field starts to be colonised by immigrant species a new
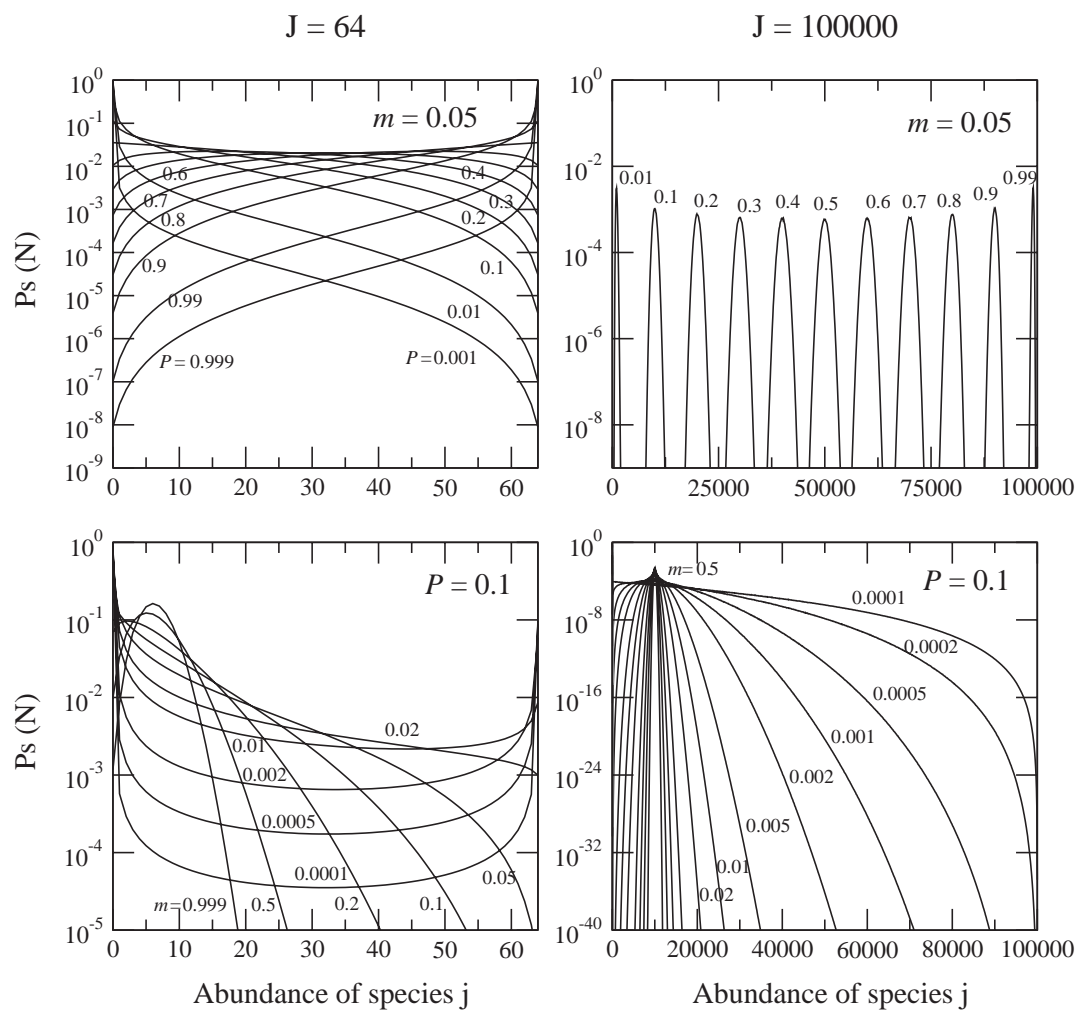

Fig. 1. Zero-sum multinomial distribution. The analytic formula (18) has been used to compute the stationary distribution, $P_{s}\left(N_{j}\right)$, for different values of the abundance of species $j$ in the metacommunity, the total number of individuals $J$ and the probability of immigration from the metacommunity, $m$. We have dropped the subscript $j$, which labels a particular species, in the figure. 
community gets formed and a pattern of species replacement develops. The transition from abandoned field to mature forest is one of the best known examples of ecological succession and is common in many places after the abandonment of agricultural land. In temperate climates, a mature forest is the end point of succession, in spite of the different potential initial conditions. The path towards the steady species-ranks distribution seems to be common to many different ecosystems (Hubbell, 2001). Furthermore, natural systems are continuously perturbed; any disturbance resumes the process of ecological succession. It is thus natural to ask: what predictions about this process can be made in the context of Hubbell's neutral theory?

In the last section it was shown that a closed form expression could be obtained for the probability of finding $N_{j}$ individuals of species $j$ in the local community when the systems has reached the stationary state. In addition to this, just mentioned, we also wish to know how the community is assembled from a given starting point. This requires us to solve for the time-dependence of the model. It is not possible, in general, to carry this out exactly, since the transition probabilities (15) and (16) are non-linear functions of $N_{j}$. It is nevertheless possible to get a very good approximation to $P\left(N_{j}, t\right)$ by using the fact that in cases of interest $J$ will be large. The approach which we will use, due to Van Kampen (1981), is rather technical and has been discussed elsewhere in some detail (Van Kampen, 1981; McKane et al., 2000), but the basic idea is quite simple. Therefore, we will avoid these complications, and quote relevant results using the correspondence with the transition probabilities (6) and (7).

The key idea is to expand about the deterministic version of the theory. In the limit where the number of individuals becomes infinite, all stochasticity is lost, and the system is completely described by a deterministic equation. This equation is not known a priori, but if it can be established, an expansion in powers of $J^{-1}$ could perhaps be set up to calculate corrections to the deterministic result which would be valid for large, but finite, $J$. Quite generally we would expect a plot of $P\left(N_{j}, t\right)$ against $N_{j}$ for fixed $t$ to be approximately Gaussian for large $J$. The motion of the peak of this distribution would move with $t$ according to the deterministic equation. Van Kampen's large $J$ expansion gives the deterministic equation as the zeroth order $(J \rightarrow \infty)$ result, with the next to leading order result giving a Gaussian distribution peaked at this value. Higher order contributions give corrections to this distribution, but they are usually so small for large $J$ that they are of very little interest. Since a Gaussian centred on a given value is completely determined by its width, there are only two things to find: (i) the deterministic equation, (ii) the width of the distribution.
In practice one writes $N_{j}=J \phi_{j}(t)+J^{1 / 2} x_{j}$, where $\phi_{j}(t)=\lim _{J \rightarrow \infty}\left(N_{j} / J\right)$ is the fraction of $j$ species which are present in the local community at time $t$ in the deterministic limit. The variable

$x_{j}=\frac{1}{\sqrt{J}}\left(N_{j}-J \phi_{j}(t)\right)$

characterises the fluctuations away from the deterministic theory. We require $\phi_{j}(t)$ and $\left\langle x_{j}^{2}\right\rangle\left(\left\langle x_{j}\right\rangle=0\right)$. Using the correspondence between the two models we obtain (McKane et al., 2000)

$\frac{d \phi_{j}}{d \tau}=m\left(P_{j}-\phi_{j}\right)$

where $\tau=t / J$ is a rescaled time. This equation is easily understood: if $\phi_{j}$ is less than the abundance of species $j$ in the regional metacommunity, then it increases. If it is more, then it decreases. The equation is easily solved to give

$\phi_{j}(\tau)=\phi_{j}(0) e^{-m \tau}+P_{j}\left(1-e^{-m \tau}\right)$.

Initially, we ask that $x_{j}(0)=0$, which means that $\phi_{j}(0)=N_{j}(0) / J=N_{j, 0} / J$. Going back to the $t$ variable gives

$\phi_{j}(t)=\frac{N_{j, 0}}{J} e^{-m t / J}+P_{j}\left(1-e^{-m t / J}\right)$.

In Hubbell (2001, Chapter 4), an alternative discretetime formulation of this local community model is given. Obviously, both time discrete and time continuous formulations give rise to the same equations for the deterministic model counterpart (Hubbell, 2001, p. 110). However, he does not address the stochastic timecontinuous formulation. Here we show that insight can be gained by finding approximate solutions to the timedependent model.

The width of the distribution can be obtained by applying Van Kampen's method. The details are given in McKane et al. (2000). It is found that

$$
\begin{aligned}
\left\langle x_{j}^{2}\right\rangle_{\tau}= & \frac{1}{m} P_{j}\left(1-P_{j}\right)\left[1-e^{-2 m \tau}\right] \\
& +\mathscr{A}_{j} \frac{2-m}{m}\left(1-2 P_{j}\right) e^{-m \tau}\left[1-e^{-m \tau}\right] \\
& -2(1-m) \mathscr{A}_{j}^{2} \tau e^{-2 m \tau},
\end{aligned}
$$

where $\mathscr{A}_{j}=\left(N_{j, 0} / J\right)-P_{j}$. We have already commented that the probability distribution is a Gaussian to the order we have been working. Specifically, in terms of the quantities calculated above

$P\left(N_{j}, t\right)=\frac{1}{\sqrt{2 \pi J\left\langle x_{j}^{2}\right\rangle_{\tau}}} \exp \left(-\frac{\left(N_{j}-J \phi_{j}(t)\right)^{2}}{2 J\left\langle x_{j}^{2}\right\rangle_{\tau}}\right)$,

where $\phi_{j}(t)$ and $\left\langle x_{j}^{2}\right\rangle_{\tau}$ are given by Eqs. (21) and (22), respectively.

In Fig. 2, we show the temporal evolution for $P\left(N_{j}, t\right)$ computed both using a Gaussian approximation 

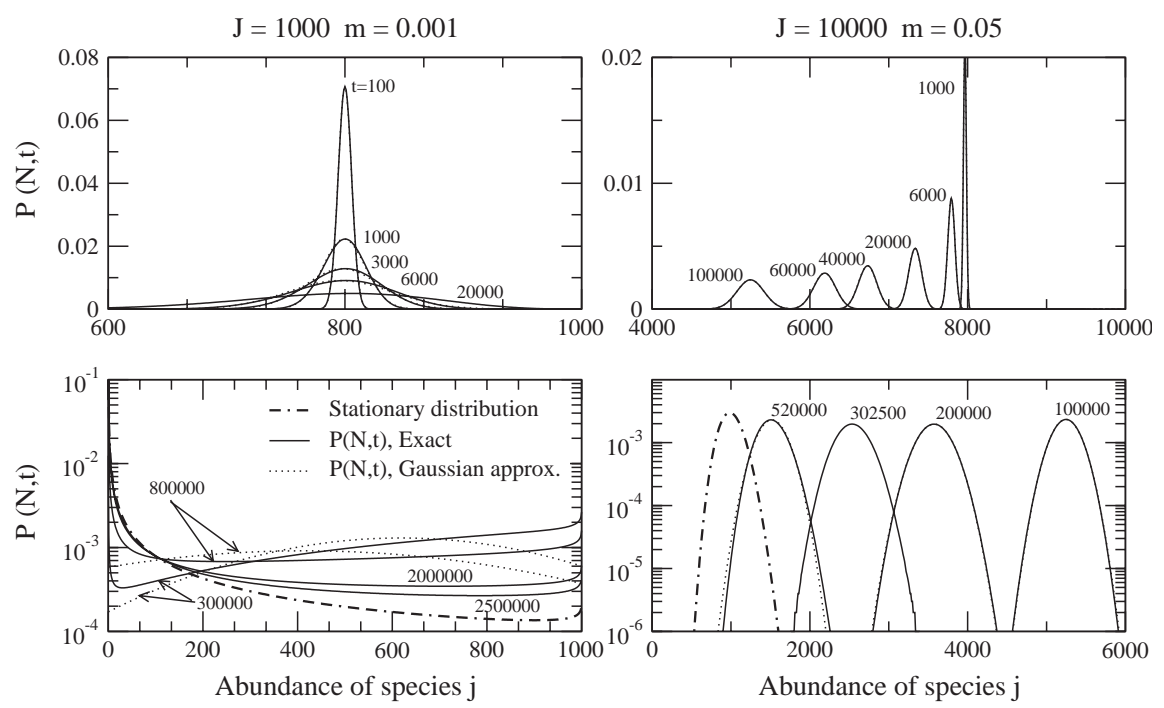

Fig. 2. Temporal evolution of the probability, $P\left(N_{j}, t\right)$, of having the $j$ th species represented by $N_{j}$ individuals. The temporal evolution has been computed using both the Gaussian approximation and the straightforward numerical integration of the exact master equation. In both cases, the initial number of individuals of the focus species was $0.8 \times J$. The relative abundance of the focus species in the metacommunity was $P_{j}=0.1$ also in both cases. We have dropped the subscript $j$, which labels a particular species, in the figure.

(Eq. (23)) and the numerical integration of the master equation. The good agreement which is obtained is a reflection of the fact that community sizes $J$ are taken to be large enough so that further terms in the large $J$ expansion are negligible. However, if the final stationary distribution does not have a Gaussian shape, more terms should be included in the expansion so as to capture the true temporal behaviour of $P\left(N_{j}, t\right)$. Notice that, while the approximation given by Eq. (23) is always represented as dotted or punctuated curves, in some cases these are not visible because they match the exact distribution so completely.

\section{Conclusion}

The main aim of this paper has been to show that aspects of Hubbell's neutral model of local community biodiversity dynamics can be solved for exactly, and even if this is not possible, calculational schemes are available which provide very good approximations to the solution. Specifically, we have shown that the stationary properties of the model, which can be obtained from the zero-sum multinomial, can all be found exactly. So, for instance, the mean value and variance of the number of individuals of species $j$, can be obtained from this probability distribution. The nature of the time evolution cannot be determined in closed form, but a controlled approximation based on assuming that the total number of individuals of all species, $J$, is large, is possible. This is an excellent approximation in most cases of interest, and we would expect that the results that we have obtained will be relevant in these situations. The applicability of our approximation scheme was checked by carrying out the numerical integration of the master equation (Eq. (10)). The results, displayed in Fig. 2, confirm our expectations.

While the results which we have reported describe the essential aspects of the solution of Hubbell's model, there are many other interesting features which are also amenable to analysis and for which definite, and wellcontrolled, results may be obtained. In general, analytical solutions provide better methods for testing these predictions using empirical data. This is particularly clear when analysing species-abundance curves. Our work can be used in a straightforward manner to improve Hubbell's numerical approach to fit special abundance data, sampling and parameter estimation (Hubbell, 2001, Chapter 9). Furthermore, since looking just at one aspect of community dynamics may not be enough to decide between two alternative models, it is of paramount importance to analytically derive the different implications of the theory. Temporal variability of species populations, species turnover, the structure of the metacommunity, and the form of the colonisation curve are examples. These, and related questions, are presently under study, and we hope to report our results in a future publication.

\section{Acknowledgments}

DA would like to thank the MACSIN research group at the UFMG, Belo Horizonte, Brazil for providing constant support and a nice working environment. This work has been supported by a grant CIRIT FI00524 
(DA) from the Catalan Government and by the Santa Fe Institute.

\section{References}

Abramowitz, M., Stegun, I.A., 1965. Handbook of Mathematical Functions. Dover, New York.

Bell, G., 2001. Neutral macroecology. Science 293, 2413-2418.

Brown, J.H., 1995. Macroecology. The University of Chicago Press, Chicago.

Caswell, H., 1976. Community structure: a neutral model analysis. Ecol. Monogr. 46, 327-354.

Ewens, W.J., 1972. The sampling theory of selectively neutral alleles. Theor. Popul. Biol. 3, 87-112.

Feller, W., 1968. An Introduction to Probability Theory and its Applications, Vol. 1, 3rd Edition. Wiley, New York.

Gardiner, C.W., 1985. Handbook of Stochastic Methods, 2nd Edition. Springer, Berlin.

Hubbell, S.P., 1997. A unified theory of biogeography and relative species abundance and its application to tropical rain forests and coral reefs. Coral Reefs 16 (Suppl.), S9-S21.
Hubbell, S.P., 2001. The Unified Theory of Biogeography and Biogeography. Princeton University Press, Princeton, NJ.

Johnson, N.L., Kotz, S., 1977. Urn Models and their Applications. Wiley, New York.

Karlin, S., McGregor, J., 1972. Addendum to a paper of W. Ewens. Theor. Popul. Biol. 3, 113-116.

Kimura, M., 1983. The Neutral Theory of Molecular Evolution. Cambridge University Press, Cambridge.

McKane, A.J., Alonso, D., Solé, R., 2000. A mean field stochastic theory for species-rich assembled communities. Phys. Rev. E 62 , 8466-8484.

Rosenzweig, M.L., 1995. Species Diversity in Space and Time. Cambridge University Press, Cambridge, UK.

Solé, R., Alonso, D., 1998. Random walks, fractals and the origins of rainforest diversity. Adv. Complex Systems 1, 203-220.

Solé, R., Alonso, D., McKane, A.J., 2000. Scaling in a network model of a multispecies ecosystem. Physica A 286, 337-344.

Solé, R., Alonso, D., McKane, A.J., 2002. Self-organized instability in complex ecosystems. Philos. Trans. R. Soc. London B 357, 667-681.

Van Kampen, N.G., 1981. Stochastic Processes in Physics and Chemistry. Elsevier, Amsterdam.

Wilson, E.O., 2003. The encyclopedia of life. Trends Ecol. Evol. 18, $77-80$ 